\title{
ANALYSIS OF LONG-TERM VARIABILITY OF CATACLYSMIC VARIABLES
}

\author{
S. Kafka ${ }^{1}$ and R. K. Honeycutt ${ }^{1}$
}

Photometric variability in cataclysmic variables (CVs) on time scales longer than a few days can be most effectively addressed by automated long-term monitoring programs such as that of RoboScope (Honeycutt \& Turner 1992): more than $100 \mathrm{CVs}$ have been monitored for about 13 years, obtaining 75 to 150 measurements per year for each system. Among the techniques being explored for analysing this data set is the use of the structure function (SF), an autocorrelation tool employed extensively for the study of the light curves of AGNs (e.g. Hufnagel \& Bregman 1992). A first order SF measures the scatter in a time series of magnitudes, $m$, as as a function of the time lag, $\tau$ (Hughes, Aller \& Aller 1992).

SF analysis can quantify variability with relative freedom from aliasing problems that are sometimes encountered in Fourier analysis. In addition, this approach has advantages when examining non-periodic data that nevertheless possess characteristic time scales. Our motivation is to define a quantitative criterion for long-term variations that are likely due to chromospheric activity cycles on the secondary star. These variations should appear quasi-periodic; however multiple cycles will be rare in our time base of 13 years. The expected signature is a downturn or flattening of the $\log (\mathrm{SF})$ vs $\log (\tau)$ plot at the characteristic time scale of the activity cycle. Here we present preliminary results. demonstrating the potential of this approach.

As a validity test of the technique, the SF of Cyg $\mathrm{X}-2$ and 3C66A (AGN) (which are included in the RoboScope program) were examined. The former is dominated by "flickering" (or 1/f) noise, and the latter has a rather typical AGN SF approaching a random walk. For the CVs, it was sometimes advantagous to remove eclipses and/or isolated VY Scl low states before calculating the SF.

The CVs examined present a variety of behaviors: a downturn at the longest time scales can be quan-
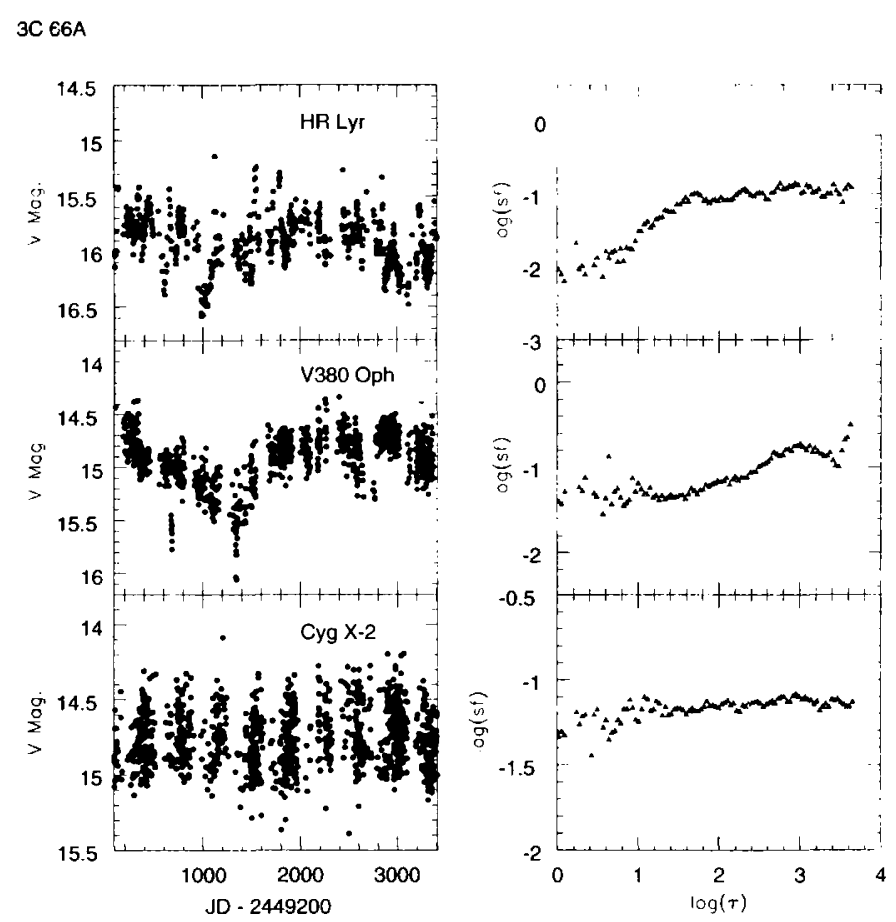

Fig. 1. Light curves and corresponding structure functions (in $m a g^{2}$ ) for HR Lyr (N Lyr 1919) and V380 Oph (NL). The related data for Cyg X-2 are also displayed for comparison. The time lag $\tau$ is measured in days.

tified as a likely measure of activity cycles. Quasiperiodicities at different $\tau$ appear as a dip in the curve. Some CVs have a rather flat SF, while others display a striking break in the slope, which indicates increasing correlation between data points at shorter time scales. Although this is still work in progress, the first results strongly encourage the use of the SF in order to reveal/describe common trends in different groups of $\mathrm{CVs}$, which will be the subject of a future discussion.

\section{REFERENCES}

Hufnagel, B. R. \& Bregman, J. N. 1992, ApJ, 386, 473 Honeycutt, R. K. \& Turner, G. W. 1992, ASP Conf. Ser. 34: Robotic Telescopes in the 1990s, 77

Hughes, P. A., Aller, H. D., \& Aller, M. F. 1992, ApJ, 396,469

\footnotetext{
${ }^{1}$ Astronomy Department, Indiana University, 319 Swain Hall West, Bloomington, IN 47405, LSA.
} 